\title{
Formononetin ameliorates IL-13-induced inflammation and mucus formation in human nasal epithelial cells by activating the SIRT1/Nrf2 signaling pathway
}

\author{
JUANJUAN HUANG, XIANFENG CHEN and AIHUA XIE \\ Department of Traditional Chinese Medicine, The Affiliated People's Hospital of \\ Ningbo University, Ningbo, Zhejiang 315040, P.R. China
}

Received March 21, 2021; Accepted June 28, 2021

DOI: $10.3892 / \mathrm{mmr} .2021 .12472$

\begin{abstract}
Formononetin has proven to be anti-inflammatory and able to alleviate symptoms of certain allergic diseases. The present study aimed to determine and elucidate the potential effects of formononetin in allergic rhinitis. JME/CF15 cells were pretreated with formononetin at different doses, followed by stimulation with IL-13. Cell Counting Kit- 8 assay was performed to determine the cytotoxicity of formononetin. The expression levels of inflammation-related proteins, histamine, IgE, TNF- $\alpha$, IL-1 $\beta$, IL- 6 , granulocyte-macrophage colony-stimulating factor and eotaxin in IL-13-stimulated JME/CF15 cells were detected using ELISAs. The expression levels of phosphorylated-NF- $\mathrm{B}$ p $65, \mathrm{NF}-\kappa \mathrm{B}$ p 65 and cyclooxygenase-2 (Cox-2) were analyzed using western blotting. Reverse transcription-quantitative PCR, western blotting and immunofluorescence were performed to measure the levels of mucin 5AC oligomeric mucus/gel-forming. Expression levels of sirtuin 1 (SIRT1) and nuclear erythroid factor 2-related factor 2 (Nrf2) proteins were also measured using western blotting. The results of the present study revealed that formononetin exerted no cytotoxic effect on the viability of JME/CF15 cells. Following stimulation of JME/CF15 cells with IL-13, formononetin suppressed the upregulated expression levels of proinflammatory cytokines. IL-13-induced formation of mucus was also attenuated by formononetin treatment. Furthermore, it was found that the SIRT1/Nrf2 signaling pathway was activated in formononetin-treated JME/CF15 cells, whereas treatment with the SIRT1 inhibitor, EX527, reversed the effects of formononetin on IL-13-induced inflammation and mucus formation in JME/CF15 cells.
\end{abstract}

Correspondence to: Dr Juanjuan Huang, Department of Traditional Chinese Medicine, The Affiliated People's Hospital of Ningbo University, 251 Baizhang East Road, Room 202, Building 6, Yinzhou, Ningbo, Zhejiang 315040, P.R. China

E-mail: huangjuanjuan20@163.com

Key words: formononetin, allergic rhinitis, sirtuin 1/nuclear erythroid factor 2-related factor 2, inflammation, mucus formation
In conclusion, the findings of the current study indicated that formononetin may activate the SIRT1/Nrf2 signaling pathway, thereby inhibiting IL-13-induced inflammation and mucus formation in JME/CF15 cells. These results suggested that formononetin may represent a promising agent for the treatment of allergic rhinitis.

\section{Introduction}

Allergic rhinitis is a non-infectious disease of the nasal mucosa, which is characterized by paroxysmal sneezing, a runny nose, nasal itching and nasal congestion (1). Patients with allergic rhinitis may have an atopic constitution, usually showing familial aggregation with some associated genetic alterations (2). With regards to external factors, exposure to allergens, such as mites, pollen, fungal spores and animal dander, often serves as the trigger for the development of allergic rhinitis $(3,4)$. Drug therapy, such as $\mathrm{H}_{1}$-antihistamines and intranasal corticosteroids, may control the symptoms of allergic rhinitis (5); however, there is currently no definitive cure (6). This disease although not fatal, does hinder general health, for example symptoms include sleep disruption and poor concentration, which can interfere with work and education $(1,7)$. Hence, medical researchers continue to search for a cure for allergic rhinitis to improve the quality of life of patients.

Formononetin is an active ingredient of a traditional Chinese herb, Radix Astragali, and may also be extracted from the inflorescence and flowered branches and leaves of the leguminous plant, Trifolium pratense L. and the whole leaf of Ononis spinosa L $(8,9)$. The anticancer properties of formononetin have been investigated in numerous studies (10-12). An increasing number of studies have also begun to focus on its potential in treating other disease types. For example, formononetin was previously found to protect against airway inflammation and oxidative stress in a mouse model of murine allergic asthma (13). Formononetin was also shown to alleviate atopic dermatitis by activating $\mathrm{G}$ protein-coupled estrogen receptors and upregulating A20 expression (14). However, to the best of our knowledge, there has been no report to date on whether formononetin exerts therapeutic effects in allergic rhinitis. Sirtuin 1 (SIRT1) is an universal gene regulator 
that affects multiple inflammation-related signaling pathways and has also been reported to play a role in attenuating ovalbumin-induced allergic symptoms in vivo (15). Upregulation of the nuclear factor erythroid 2-related factor 2 (Nrf2)/heme oxygenase (HO)-1 signaling pathway by mangiferin was previously demonstrated to be associated with the improvement of the ovalbumin-induced inflammatory response in mice with allergic rhinitis (16). Moreover, the regulatory effect of formononetin on SIRT1 expression has already been identified in various types of disease. For example, formononetin was shown to ameliorate cholestasis by regulating hepatic SIRT1 and peroxisome proliferator-activated receptor $\alpha$ expression (17).

IL-13 is a typical T helper cell 2 cytokine and serves a prominent role in the regulation of numerous types of allergic disease by activating its receptor and the associated STAT6 (18). IL-13 is often used to simulate pathological changes observed in a number of airway allergic diseases, such as eosinophil recruitment, mucus cell metaplasia, subepithelial fibrosis and smooth muscle hypertrophy (19). On this basis, IL-13 induction was used in the present study to establish an in vitro allergic rhinitis model.

The present study hypothesized that formononetin may exert protective effects in an allergic rhinitis model established with IL-13 via regulating the SIRT1/Nrf2 signaling pathway to inhibit inflammatory cytokine secretion and mucus formation. The present study was undertaken to verify this hypothesis, with the aim of providing a novel agent for the effective treatment or alleviation of allergic rhinitis.

\section{Materials and methods}

Cell culture and treatment. The JME/CF15 human nasal epithelial cell line was acquired from The Cell Bank of Type Culture Collection of The Chinese Academy of Sciences. The cells were cultured in DMEM/F12 (cat. no. 11320033; Gibco; Thermo Fisher Scientific, Inc.) supplemented with 10\% FBS (cat. no. 12484010; Gibco; Thermo Fisher Scientific, Inc.), 2 mM L-glutamine (cat. no. A2916801; Gibco; Thermo Fisher Scientific, Inc.), 100 U/ml penicillin (cat. no. V900929; Sigma-Aldrich; Merck KGaA) and $100 \mathrm{mg} / \mathrm{ml}$ streptomycin (cat. no. HY-B0472; MedChemExpress). The cells were maintained in $95 \%$ air and $5 \% \mathrm{CO}_{2}$ at $37^{\circ} \mathrm{C}$.

The allergic rhinitis model was established by stimulating the JME/CF15 cells with $10 \mathrm{ng} / \mathrm{ml}$ IL-13 (cat. no. HY-P7033; MedChemExpress) at $37^{\circ} \mathrm{C}$ for $30 \mathrm{~min}$ as previously described (20), or pretreatment with formononetin [cat. no. CN00152; ChemeGen (Shanghai) Biotechnology Co., Ltd.] at doses of $0.1,1$ and $10 \mu \mathrm{M}(14,21)$ or EX527 (SIRT1 inhibitor; cat. no. E7034; Sigma-Aldrich; Merck KGaA) at a dose of $10 \mu \mathrm{M}$ at $37^{\circ} \mathrm{C}$ for $24 \mathrm{~h}$ (22). Normal JME/CF15 cells without any treatment were used as the model group.

Cell Counting Kit-8 (CCK-8) assay. The cytotoxicity of formononetin was detected using a CCK-8 assay (cat. no. C0037; Beyotime Institute of Biotechnology) as previously described (23). Briefly, $100 \mu \mathrm{l}$ cell suspension was added into a 96-well plate using a density of $2 \times 10^{3}$ cells/well, with incubation at $37^{\circ} \mathrm{C}$ for $24 \mathrm{~h}$. Cells were then treated accordingly, and after $24 \mathrm{~h}, 10 \mu \mathrm{l}$ CCK-8 solution was added to each well.
Subsequently, the cells were incubated with CCK- 8 solution at $37^{\circ} \mathrm{C}$ for $1.5 \mathrm{~h}$. The absorbance was measured at a wavelength of $450 \mathrm{~nm}$ using a microplate reader.

ELISA. ELISAs were performed to examine the proinflammatory cytokine secretion in IL-13-stimulated JME/CF15 cells as previously described (24). The levels of histamine, IgE, TNF- $\alpha$, IL-1 $\beta$, IL-6, granulocyte-macrophage colony-stimulating factor (GM-CSF) and eotaxin were detected using histamine (cat. no. ab213975; Abcam), IgE (cat. no. ab195216; Abcam), TNF- $\alpha$ (cat. no. PT518; Beyotime Institute of Biotechnology), IL-1 $\beta$ (cat. no. PI305; Beyotime Institute of Biotechnology), IL-6 (cat. no. PI330; Beyotime Institute of Biotechnology) ELISA kits, GM-CSF (cat. no. PG355; Beyotime Institute of Biotechnology) and eotaxin (cat. no. PC115; Beyotime Institute of Biotechnology), respectively. The optical density value was measured at a wavelength of $450 \mathrm{~nm}$ using an ELISA detector.

Western blotting. Whole cell protein extracts were extracted using RIPA lysis buffer, while nuclear and cytoplasmic proteins were extracted using Nuclear and Cytoplasmic Protein Extraction Kit (cat. no. P0027; Beyotime Institute of Biotechnology), and all protein samples were quantified using the BCA method. The sample proteins $(20 \mu \mathrm{g} / \mathrm{lane})$ were separated via $10 \%$ SDS-PAGE and subsequently transferred onto PVDF membranes. The membrane was removed from the transfer apparatus and soaked in TBS with $10 \%$ Tween-20 (TBST) washing buffer (Shanghai Aladdin Biochemical Technology Co., Ltd.) twice for $15 \mathrm{~min}$ each time. The membrane was then blocked with $10 \%$ non-fat dried milk freshly diluted in TBST and rocked on a rotating shaker for $15 \mathrm{~min}$ at room temperature. After rinsing with TBST, the membranes were incubated with the following primary antibodies diluted in $1 \%$ non-fat dried milk at $4^{\circ} \mathrm{C}$ overnight: Anti-phosphorylated (p)-NF- $\mathrm{B}$ p65 (1:1,000; cat. no. ab239882; Abcam), anti-NF-кB p65 (1:1,000; cat. no. ab207297; Abcam), anti-cyclooxygenase 2 (Cox-2; 1:1,000; cat. no. 12282; Cell Signaling Technology, Inc.), anti-mucin 5AC oligomeric mucus/gel-forming (MUC5AC; 1:20,000; cat. no. ab198294; Abcam), anti-Nrf2 (1:1,000; cat. no. ab62352; Abcam), anti-SIRT1 (1:1,000; cat. no. ab189494; Abcam), anti- $\beta$-actin (1:1,000; cat. no. ab8227; Abcam) and anti-lamin B1 (1:1,000; cat. no. ab229025; Abcam). Following the primary antibody incubation, the membranes were rinsed three times in TBST and incubated with a HRP-conjugated secondary antibody (1:2,000; cat. no. ab97051; Abcam) diluted in 1\% non-fat dried milk for $30 \mathrm{~min}$ at room temperature. Excess secondary antibody was then removed from the membrane with three rinses in $20 \mathrm{ml}$ TBST for $5 \mathrm{~min}$ each time. Protein bands were analyzed using a standard chemiluminescence detection reagent (cat. no. P0018S; Beyotime Institute of Biotechnology) and ImageJ software (version $1.48 \mathrm{v}$; National Institutes of Health) was used for semi-quantification as previously described (23).

Reverse transcription-quantitative PCR (RT-qPCR). mRNA expression levels of MUC5AC in IL-13-stimulated JME/CF15 cells were detected using RT-qPCR as previously described (23). Total RNA from JME/CF15 cells was extracted using TRIzol ${ }^{\circledR}$ reagent (cat. no. 15596018; Invitrogen; Thermo 
Fisher Scientific, Inc.) and the quality was assessed using a NanoDrop 2000 spectrophotometer (Thermo Fisher Scientific, Inc.). Total RNA was reverse transcribed into cDNA using a Sensiscript RT kit (cat. no. 205213; Qiagen, Inc.) according to the manufacturer's protocol. qPCR was subsequently performed using a TaqMan Universal Master mix II (cat. no. 4440048; Applied Biosystems; Thermo Fisher Scientific, Inc.) on an ABI Real-Time PCR system (Applied Biosystems; Thermo Fisher Scientific, Inc.). The following thermocycling conditions were used for the qPCR: Initial denaturation for $3 \mathrm{~min}$ at $95^{\circ} \mathrm{C}$; followed by 40 cycles of denaturation for $15 \mathrm{sec}$ at $95^{\circ} \mathrm{C}$, and annealing and extension for $30 \mathrm{sec}$ at $60^{\circ} \mathrm{C}$. The primers used for the qPCR were as follows: MUC5AC forward, 5'-GCTTCCTGCTCCGAGATGT-3' and reverse, 5'-AAGACG CAGCCCTCATAGAA-3'; and GAPDH forward, 5'-CACCCA CTCCTCCACCTTTG-3' and reverse, 5'-CCACCACCCTGT TGCTGTAG-3'. The relative expression levels were calculated using the $2^{-\Delta \Delta \mathrm{Cq}}$ method (25).

Immunofluorescence (IF). IF was employed to further verify the expression of MUC5AC in IL-13-stimulated JME/CF15 cells as previously described (26). Briefly, following fixation with 4\% paraformaldehyde (Shanghai Aladdin Biochemical Technology Co., Ltd.) for $10 \mathrm{~min}$ at room temperature, the cells were incubated with $0.5 \%$ Triton X-100 (cat. no. HFH10; Invitrogen; Thermo Fisher Scientific, Inc.) at $25^{\circ} \mathrm{C}$ for $20 \mathrm{~min}$. Subsequently, 5\% BSA (cat. no. B265993; Shanghai Aladdin Biochemical Technology Co., Ltd.) diluted in PBS (Beyotime Institute of Biotechnology) was used to block the cells at room temperature for $1 \mathrm{~h}$ before incubation with an anti-MUC5AC antibody $(1: 250)$ overnight at $4^{\circ} \mathrm{C}$. Following the primary antibody incubation, the cells were washed with PBS and incubated with a FITC-conjugated goat anti-rabbit IgG secondary antibody (1:200; cat. no. P0186; Beyotime Institute of Biotechnology) at $25^{\circ} \mathrm{C}$ for $1 \mathrm{~h}$, followed by staining with DAPI (MedChemExpress) for $15 \mathrm{~min}$ at room temperature. Stained cells were visualized using a fluorescence microscope (magnification, x200).

Statistical analysis. Independent experiments were performed at least three times. The data are presented as the mean \pm SD and were analyzed using a one-way ANOVA followed by a Tukey's post hoc test. GraphPad Prism 6 software (GraphPad Software, Inc.) was used for the statistical analysis. $\mathrm{P}<0.05$ was considered to indicate a statistically significant difference.

\section{Results}

Formononetin does not affect JME/CF15 cell viability. The cytotoxicity of formononetin was examined using a CCK-8 assay, which demonstrated that the viability of JME/CF15 cells was not significantly altered following formononetin treatment at doses of $0.1,1$ or $10 \mu \mathrm{M}$ (Fig. 1). These results suggested that formononetin may exert no cytotoxic effect on JME/CF15 cells.

Formononetin inhibits inflammatory cytokine secretion in IL-13-stimulated JME/CF15 cells. According to the results of the ELISAs (Fig. 2A and B), the levels of the immunoregulatory autacoid histamine, the allergy index $\operatorname{IgE}$ and the

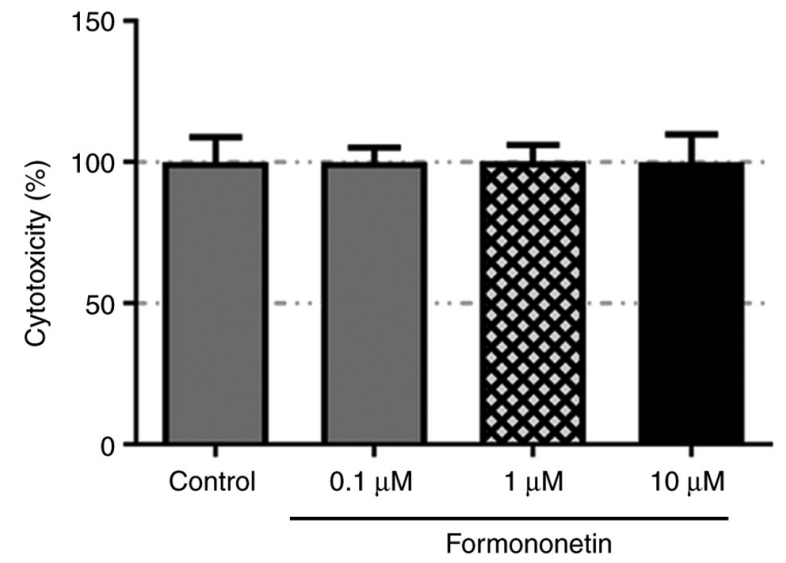

Figure 1. Formononetin has no impact on JME/CF15 cell viability. Cytotoxicity of formononetin in JME/CF15 cells was detected using a Cell Counting Kit-8 assay.

proinflammatory cytokines, TNF- $\alpha$, IL-1 $\beta$ and IL-6, were significantly upregulated in IL-13-stimulated JME/CF15 cells compared with the control group, and were subsequently downregulated in a dose-dependent manner following pretreatment with $0.1,1$ or $10 \mu \mathrm{M}$ formononetin. Furthermore, IL-13 stimulation significantly elevated the levels of GM-CSF and eotaxin in JME/CF15 cells compared with the control group, which were then dose-dependently reduced by formononetin treatment at all doses (Fig. 2C). Western blotting revealed that the expression levels of inflammation-related Cox-2 and p-NF- $\mathrm{B}$ p65/NF- $\mathrm{B}$ p65 were significantly upregulated in IL-13-stimulated JME/CF15 cells compared with the control group, while their expression levels were subsequently downregulated following formononetin treatment (Fig. 2D). These results suggested an inhibitory effect of formononetin on the secretion of inflammatory cytokines in IL-13-stimulated JME/CF15 cells.

Formononetin inhibits mucus formation in IL-13-stimulated $J M E / C F 15$ cells. Overexpression of human MUC5AC has been reported to contribute to the formation of mucus in airway inflammation $(27,28)$; therefore, its protein expression levels were detected in the allergic rhinitis model in the present study. It was observed that, while IL-13 induced high expression of MUC5AC, pretreatment with formononetin effectively reduced MUC5AC expression levels in IL-13-stimulated JME/CF15 cells (Fig. 3A and B). Formononetin at $10 \mu \mathrm{M}$ was associated with the most significant reduction in MUC5AC expression and was thus selected for use in subsequent experiments. As shown in Fig. 3C, the fluorescence intensity of MUC5AC was increased in the IL-13 group compared with the control group, while it was markedly weakened in the IL-13 + formononetin group. These results indicated that formononetin may prevent the formation of mucus in IL-13-stimulated JME/CF15 cells.

Formononetin activates the SIRT1/Nrf2 signaling pathway. To understand the mechanism of action of formononetin, it was investigated whether there was an interaction between formononetin and the SIRT1/Nrf2 signaling pathway. Western blotting revealed that the expression levels of SIRT1 and nucleic Nrf2 were downregulated in IL-13-stimulated JME/CF15 cells compared with the control group, and were 
A

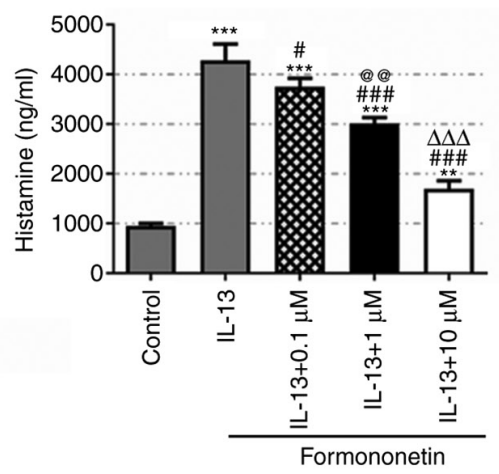

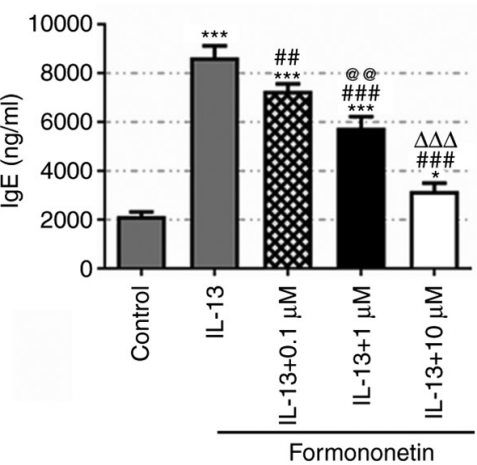

B
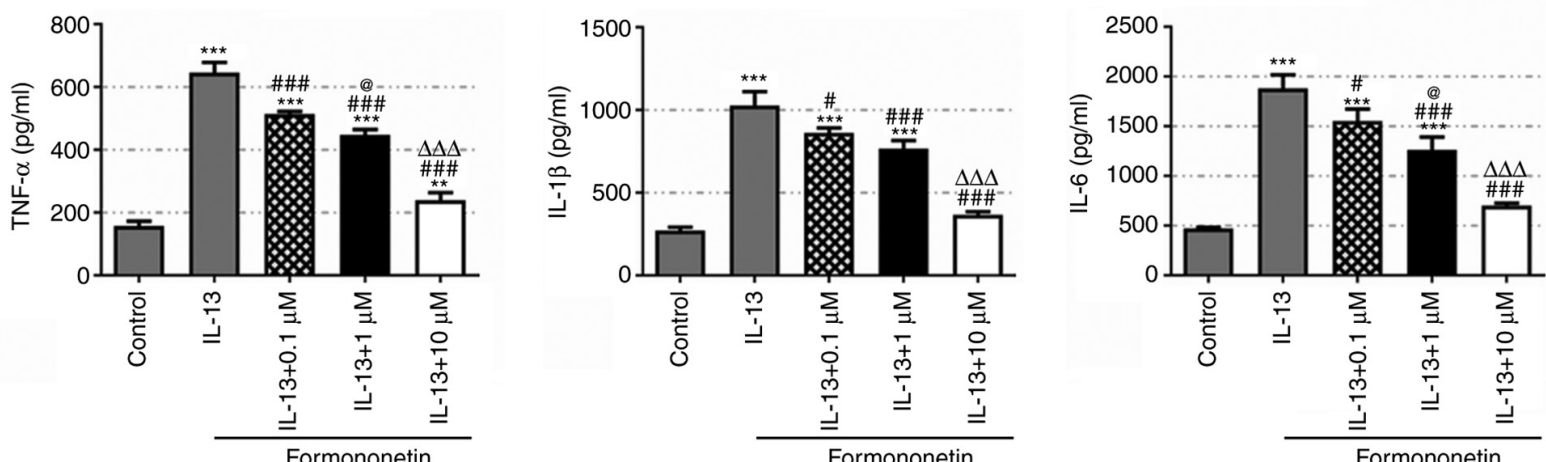

C

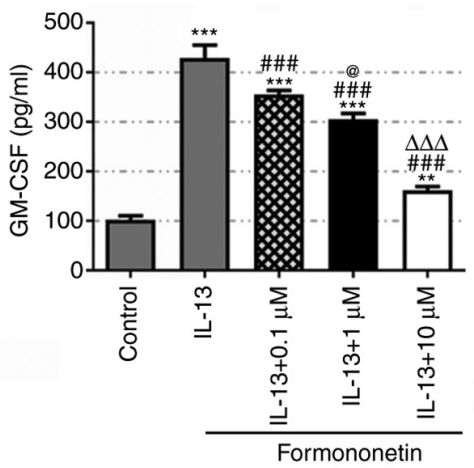

Formononetin

Formononetin

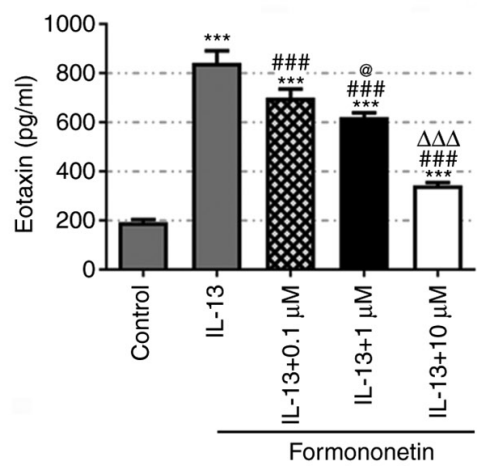

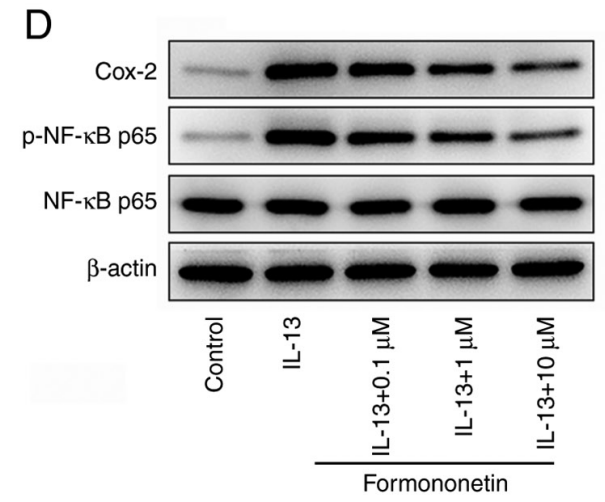

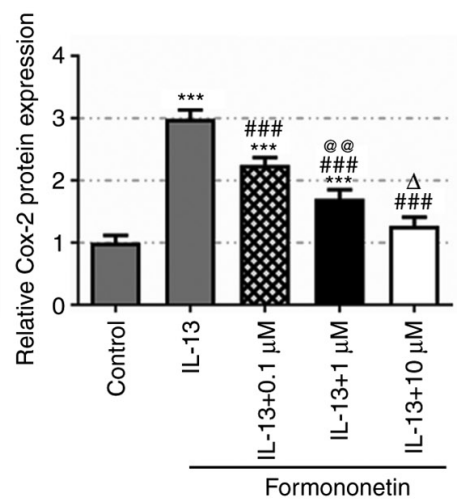

Figure 2. Formononetin inhibits inflammatory cytokine secretion in IL-13-stimulated JME/CF15 cells. Concentrations of (A) histamine and IgE, (B) TNF- $\alpha$, IL-1 $\beta$ and IL-6 and (C) GM-CSF and eotaxin in IL-13-stimulated JME/CF15 cells treated with 0, 0.1, 1 or $10 \mu$ M formononetin were detected using ELISAs.

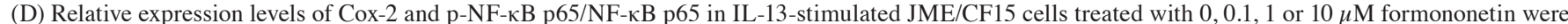
detected using western blotting. " $\mathrm{P}<0.05,{ }^{* *} \mathrm{P}<0.01,{ }^{* * *} \mathrm{P}<0.001$ vs. control; ${ }^{\#} \mathrm{P}<0.05,{ }^{\# / t} \mathrm{P}<0.01,{ }^{\# \# \#} \mathrm{P}<0.001$ vs. IL-13; ${ }^{\circledR} \mathrm{P}<0.05,{ }^{\circledR @} \mathrm{P}<0.01$ vs. IL-13 + $0.1 \mu \mathrm{M}$; ${ }^{\Delta} \mathrm{P}<0.05,{ }^{\Delta \Delta} \mathrm{P}<0.001$ vs. IL-13 $+1 \mu \mathrm{M}$. GM-CSF, granulocyte-macrophage colony-stimulating factor; Cox-2, cyclooxygenase 2; $\mathrm{p}-$, phosphorylated.

subsequently dose-dependently upregulated following treatment with formononetin (Fig. 4). Conversely, the expression levels of cytoplasmic Nrf2 exhibited the opposite trends. These findings indicated that formononetin may activate the SIRT1/Nrf2 signaling pathway.
SIRT1 inhibitor EX527 reverses the effects of formononetin on IL-13-stimulated JME/CF15 cells. To verify the role of the SIRT1/Nrf2 axis in the mechanism of action of formononetin, the expression levels of SIRT1 in IL-13-stimulated JME/CF15 cells were inhibited using EX527. A significant rise in the 
B
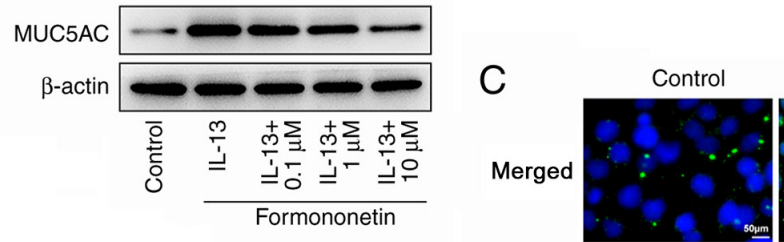

$\mathrm{IL}-13$

IL-13+Formononetin
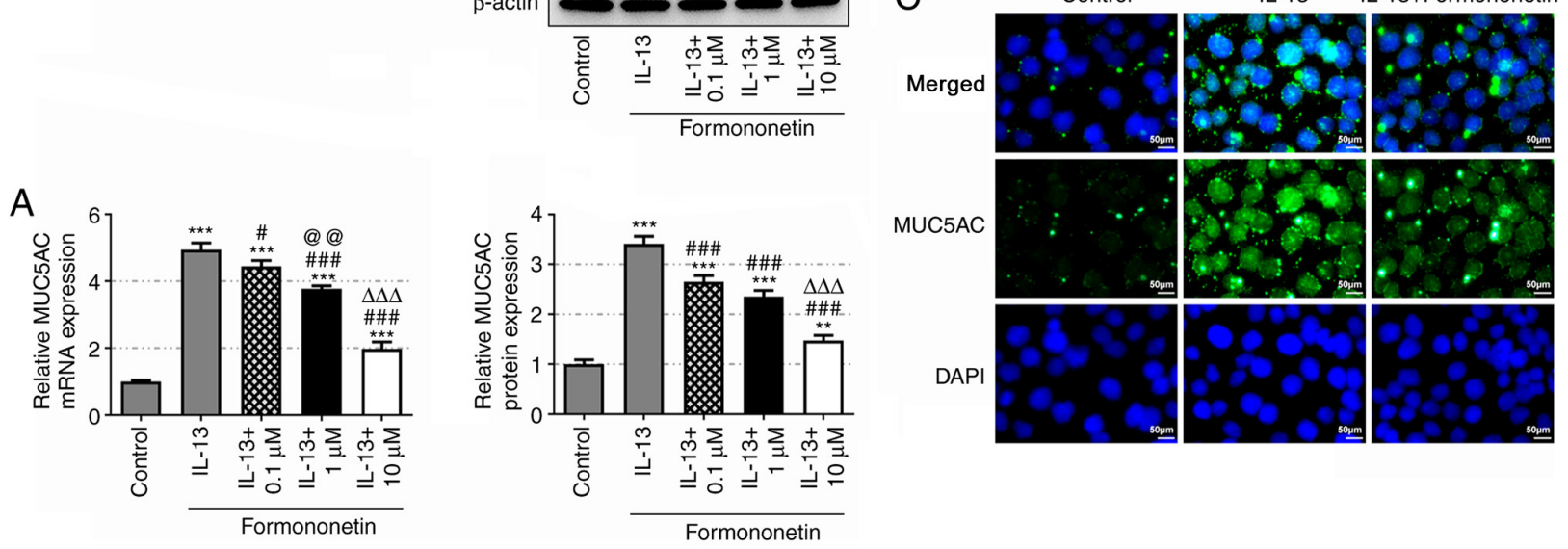

Figure 3. Formononetin inhibits mucus formation in IL-13-stimulated JME/CF15 cells. Relative mRNA and protein expression levels of MUC5AC in IL-13-stimulated JME/CF15 cells treated with $0,0.1,1$ or $10 \mu \mathrm{M}$ formononetin were detected using (A) reverse transcription-quantitative PCR and (B) western blotting, respectively. ${ }^{* *} \mathrm{P}<0.01,{ }^{* * *} \mathrm{P}<0.001$ vs. control; ${ }^{\# \mathrm{P}}<0.05$, ${ }^{\# \# \mathrm{P}}<0.001$ vs. IL-13; ${ }^{\circledR}{ }^{\circledR} \mathrm{P}<0.01$ vs. IL-13 $+0.1 \mu \mathrm{M} ;{ }^{\Delta \Delta \Delta} \mathrm{P}<0.001$ vs. IL-13 + $1 \mu \mathrm{M}$. (C) Fluorescence intensity of MUC5AC in JME/CF15 cells treated with control, IL-13 or IL-13 + formononetin (10 $\mu \mathrm{M})$ was detected using immunofluorescence. MUC5AC is shown as the green fluorescence Scale bar, $50 \mu \mathrm{m}$. MUC5AC, mucin 5AC oligomeric mucus/gel-forming.
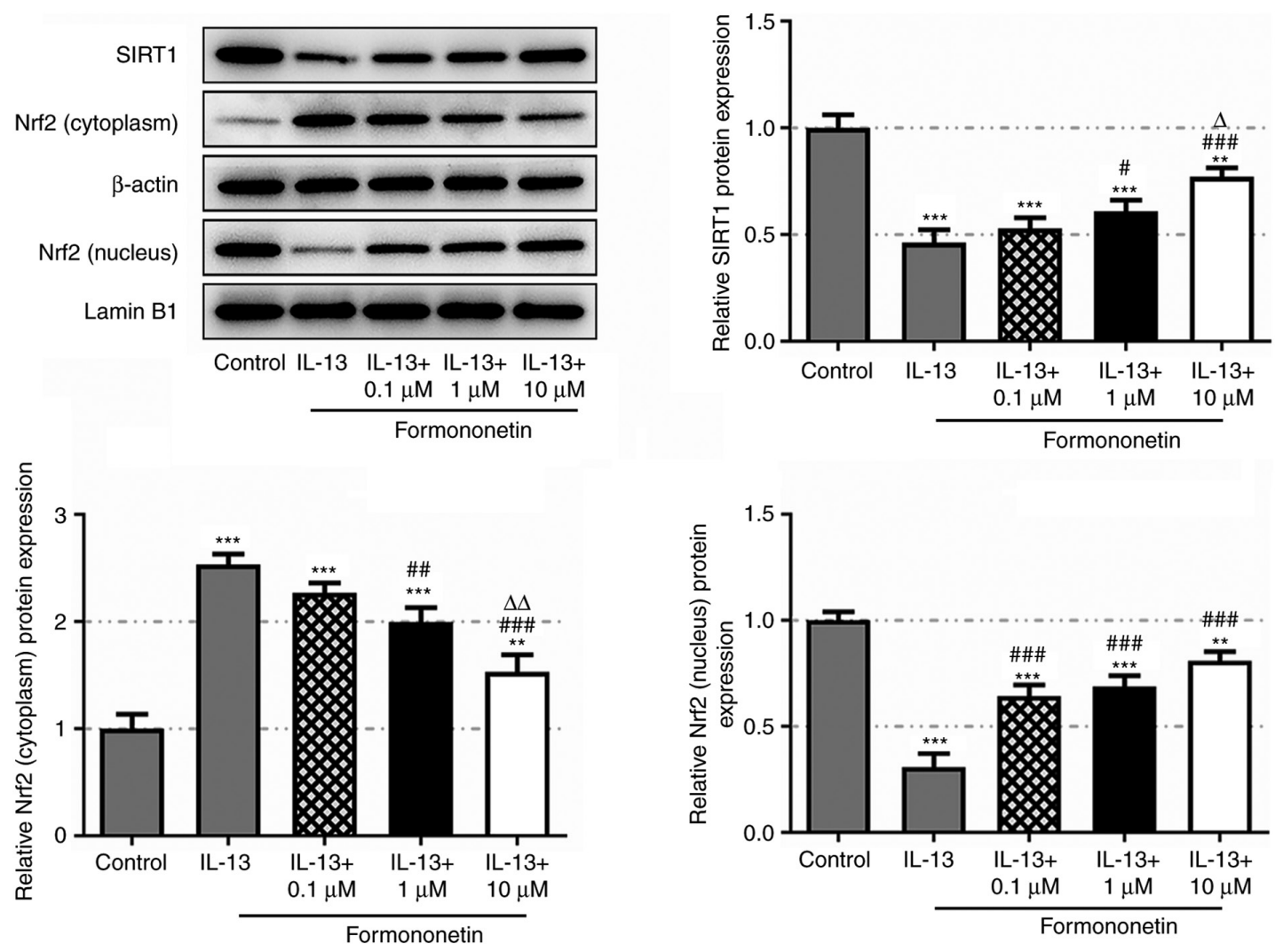

Figure 4. Formononetin activates the SIRT1/Nrf2 signaling pathway. Relative protein expression levels of SIRT1, and cytoplasmic and nucleic Nrf2 in IL-13-stimulated JME/CF15 cells treated with $0,0.1,1$ or $10 \mu \mathrm{M}$ formononetin were detected using western blotting. ${ }^{* * *} \mathrm{P}<0.01,{ }^{* * * *} \mathrm{P}<0.001 \mathrm{vs}$. control; ${ }^{\mathrm{P}} \mathrm{P}<0.05$, ${ }^{\# \prime} \mathrm{P}<0.01,{ }^{\# \# \#} \mathrm{P}<0.001$ vs. IL-13; ${ }^{{ }^{2}} \mathrm{P}<0.05,{ }^{\Delta \Delta} \mathrm{P}<0.01$ vs. IL-13 $+1 \mu \mathrm{M}$. SIRT1, sirtuin 1; Nrf2, nuclear factor erythroid 2-related factor 2.

levels of histamine, IgE, TNF- $\alpha$, IL-1 $\beta$ and IL- 6 was observed in IL-13-stimulated formononetin-treated JME/CF15 cells following SIRT1 inhibition (Fig. 5A and B). EX527 treatment also increased the levels of GM-CSF and eotaxin, which were suppressed by formononetin treatment in IL-13-stimulated JME/CF15 cells (Fig. 5C). Furthermore, while the expression

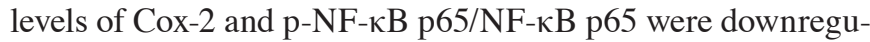
lated by formononetin treatment in IL-13-stimulated JME/CF15 cells, inhibiting SIRT1 with EX527 partially reversed their expression levels (Fig. 5D). The expression levels of nucleic Nrf2 were downregulated, while the expression levels of cytoplasmic Nrf2 were upregulated in IL-13-stimulated JME/CF15 

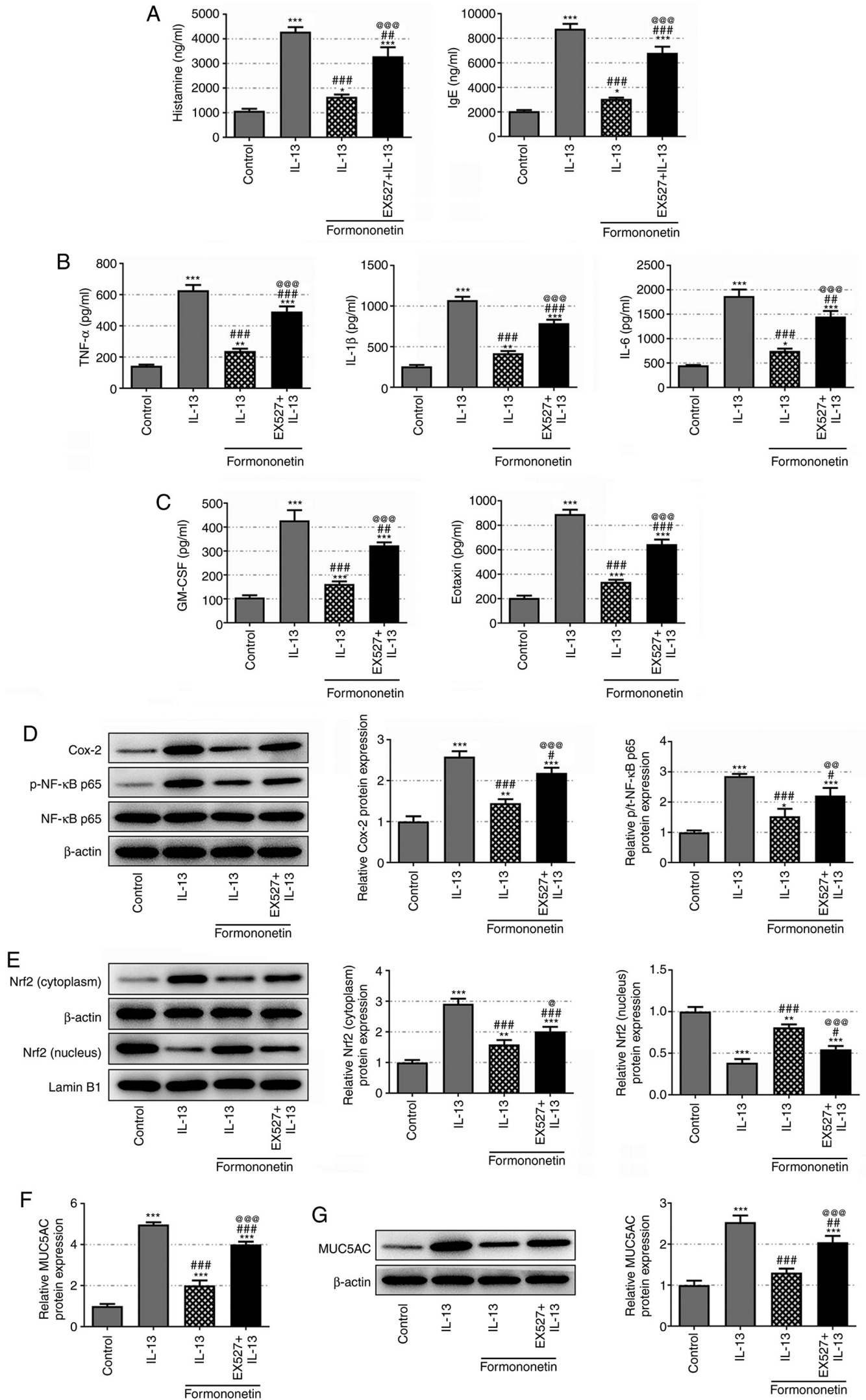

Figure 5. Sirtuin 1 inhibitor EX527 reverses the effects of formononetin on IL-13-stimulated JME/CF15 cells. Concentrations of (A) histamine and IgE, (B) TNF- $\alpha$, IL-1 $\beta$ and IL-6 and (C) GM-CSF and eotaxin in JME/CF15 cells treated with IL-13, IL-13 + formononetin or IL-13 + formononetin + EX527 were detected using ELISAs. (D) Relative protein expression levels of Cox-2 and p-NF-kB p65/NF-кB p65 in JME/CF15 cells treated with IL-13, IL-13 + formononetin or IL-13 + formononetin + EX527 were detected using western blotting. (E) Relative protein expression levels of nucleic and cytoplasmic Nrf2 in JME/CF15 cells treated with IL-13, IL-13 + formononetin or IL-13 + formononetin + EX527 were detected using western blotting. Relative mRNA and protein expression levels of MUC5AC in JME/CF15 cells treated with IL-13, IL-13 + formononetin or IL-13 + formononetin + EX527 were detected using (F) reverse transcription-quantitative PCR and $(\mathrm{G})$ western blotting, respectively. ${ }^{*} \mathrm{P}<0.05,{ }^{* *} \mathrm{P}<0.01,{ }^{* * *} \mathrm{P}<0.001$ vs. control; ${ }^{\# \mathrm{P}}<0.05$, ${ }^{\# \#} \mathrm{P}<0.01,{ }^{\# \# \#} \mathrm{P}<0.001$ vs. IL-13; ${ }^{\circledR} \mathrm{P}<0.05,{ }^{\circledR} \mathrm{P}<0.01,{ }^{@} @{ }^{\circledR} \mathrm{P}<0.001$ vs. IL-13 + formononetin. GM-CSF, granulocyte-macrophage colony-stimulating factor; Cox-2, cyclooxygenase 2; p-, phosphorylated; Nrf2, nuclear factor erythroid 2-related factor 2; MUC5AC, mucin 5AC oligomeric mucus/gel-forming. 
cells. After pretreatment with formononetin, the expression level of nucleic Nrf2 was upregulated and cytoplasmic Nrf2 expression was downregulated, which was partially reversed following EX527 treatment (Fig. 5E). Similarly, EX527 treatment rescued the expression levels of MUC5AC, which were downregulated by formononetin treatment in IL-13-stimulated JME/CF15 cells (Fig. 5F and G). These results indicated that formononetin may inhibit the secretion of proinflammatory cytokines and mucus formation in IL-13-stimulated JME/CF15 cells by activating the SIRT1/Nrf2 signaling pathway.

\section{Discussion}

Allergic rhinitis, which currently affects $\sim 40 \%$ of the world population, is a global health concern that is associated with a notable socioeconomic burden and may lead to serious complications, including asthma and otitis media (29). Allergic rhinitis is an atopic disorder of the nasal mucosa that is mainly characterized by IgE-mediated histamine release and involves a variety of immunocompetent cells and cytokines, such as pathogenic memory T helper 2 cells, IL-4, IL-5 and IL-13, which are triggered following exposure of the atopic individual to allergens $(30,31)$. Risk factors for allergic rhinitis are present at all ages $(32)$, and include genetic factors $(33,34)$, environmental pollution (35) and environmental allergen exposure (36). The symptoms of the disease may be effectively controlled through standardized treatment (examples include treatment with intranasal corticosteroids, H1-antihistamine and leukotriene receptor antagonist) (5) and care (37); however, long-term treatment poses a heavy economic burden (38).

Formononetin, a type of phytoestrogen derived from the medicinal plant Trifolium pretense L., has been proven to be an active component with promising anticancer properties, as evidenced by previous research $(39,40)$. In addition to its anticancer properties, formononetin has also been found to exert antioxidant and anti-inflammatory effects in various types of disease. For example, previous studies have reported that formononetin played a role in preventing allergic diseases and that it could effectively attenuate atopic dermatitis through regulation of G-protein-coupled estrogen receptor and A20 expression $(14,41)$. It was also shown that formononetin regulated $\mathrm{NF}-\kappa \mathrm{B}$ signaling to inhibit the inflammatory response and promote the angiogenesis of the gastric mucosa in mice with gastric ulcers (42). However, to the best of our knowledge, whether formononetin has a beneficial effect on allergic rhinitis has yet to be reported. Excessive release of histamine induced by allergen exposure is commonly known to be an active contributor to the occurrence of allergic rhinitis (43). $\mathrm{IgE}$ is an important mediator of the endogenous release of histamine, the level of which is also closely associated with asthma, food allergies and allergic rhinitis (44-47). It was previously demonstrated that formononetin decreased the levels of histamine and proinflammatory cytokines to alleviate allergic inflammation (21). In the present study, a cellular model of allergic rhinitis was established using IL-13 stimulation, in which high levels of histamine, $\operatorname{IgE}$ and proinflammatory cytokines, TNF- $\alpha$, IL-1 $\beta$ and IL-6, were detected. However, treatment with formononetin markedly decreased these levels in a dose-dependent manner. Previous studies have reported high concentrations of GM-CSF in patients with nasal allergy, which was also found to be associated with aggravated rhinorrhea $(48,49)$. A more recent study also demonstrated the value of GM-CSF in assessing chronic rhinitis due to its positive correlation with eosinophilic inflammation (50). The results of the present study revealed high levels of both GM-CSF and eotaxin in IL-13-stimulated JME/CF15 cells, while treatment with formononetin was demonstrated to effectively suppress GM-CSF and eotaxin levels. Upregulated Cox-2 expression has been proven to be a cancer-related biomarker and is also closely associated with inflammation $(51,52)$. Some of the anti-inflammatory effects of formononetin were discovered to be mediated through its suppression of Cox-2 expression in neurological lesions and kidney injury, among others $(53,54)$. NF- $\kappa \mathrm{B}$ p65 is a member of the NF- $\mathrm{NB}$ family that acts as a master regulator of inflammation and is widely found in animal cells (55). Formononetin was reported to inactivate NF- $\mathrm{NB}$ p65 to obstruct osteoclastogenesis and protect against liver injury $(56,57)$. Consistent with previous findings, downregulated expression levels of Cox-2 and p-NF- $\kappa$ B p65 were also found in the present study following formononetin treatment in IL-13-stimulated JME/CF15 cells. Taken together, these changes suggested that formononetin may inhibit IL-13-induced proinflammatory cytokine secretion in JME/CF15 cells.

MUC5AC is a gel-forming mucin that is normally present in human airways, the high expression of which leads to the oversecretion of mucus and was recently identified in the airway mucus of patients with severe coronavirus-19 disease (58). In the present study, IL-13 significantly upregulated the protein expression levels of MUC5AC in JME/CF15 cells, which were markedly suppressed following formononetin treatment, as evidenced by the results of the RT-qPCR, western blotting and IF experiments. These findings indicated that formononetin may inhibit mucus formation in allergic rhinitis.

In addition to the effects of formononetin on allergic rhinitis, the mechanism underlying its effects was also investigated. SIRT1 is a well-known regulator of chronic inflammatory responses, which has been investigated in the context of the alleviation of asthma as well as allergic rhinitis $(15,59)$. Wu et al (60) found that SIRT1 activation upregulated Nrf2 expression and inhibited the $\mathrm{NF}-\kappa \mathrm{B}$ signaling pathway to relieve sepsis-induced inflammation and apoptosis. Furthermore, it was demonstrated in a previous study that formononetin served as a SIRT1 activator and improved diabetic neuropathy (61). In addition, formononetin was found to upregulate SIRT1 expression and inhibit lipopolysaccharide-induced high mobility group box 1 release, thereby inhibiting the cellular inflammatory response (62). SIRT1 activated by resveratrol could also alleviate ovalbumin-induced allergic rhinitis in mice (63). SIRT1 knockdown alleviated the symptoms and inflammatory indicators of allergic rhinitis in HIF1 $\alpha$ CD $11 c^{-/-}$mice (64). Activation of the Nrf2/HO-1 signaling pathway could suppress allergic rhinitis activity (65). In addition, mangiferin attenuated allergic rhinitis by enhancing the Nrf2/HO-1 signaling pathways (16). The present study revealed that formononetin may activate the SIRT1/Nrf2 signaling pathway. Normally, Nrf2 and Kelch like ECH associated protein (Keap1) bind as a dimer in the cytoplasm, and when stimulated, Nrf2 dissociates from Keap1 and translocates into the nucleus, thereby inducing the expression of encoded related enzymes, such as 
HO-1 (66). In the present study, formononetin was shown to upregulate the expression of $\mathrm{Nrf} 2$ in the nucleus and promote the nuclear translocation of Nrf2, and thus exert an effect. Moreover, inhibiting SIRT1 with EX527 largely reversed the effects of formononetin on IL-13-induced inflammation and mucus secretion in JME/CF15 cells, and reduced the nuclear translocation of Nrf2, further verifying the role of SIRT1/Nrf2 signaling in the mechanism of action of formononetin. However, the present conclusion is based solely on cell experiments and further animal experiments should be conducted to validate the current study findings.

In conclusion, the findings of the present study provided evidence to suggest that formononetin may ameliorate IL-13-induced proinflammatory cytokine secretion and mucus formation in JME/CF15 cells by activating the SIRT1/Nrf2 signaling pathway. These findings are significant as they indicate the curative potential of formononetin in the treatment of allergic rhinitis, and it may be considered as a novel therapeutic strategy for this disease.

\section{Acknowledgements}

Not applicable.

\section{Funding}

No funding was received.

\section{Availability of data and materials}

The datasets used and/or analyzed during the current study are available from the corresponding author on reasonable request.

\section{Authors' contributions}

$\mathrm{JH}$ conceived and designed the experiments in the present study. JH, XC and AX performed the experiments. JH and $\mathrm{XC}$ analyzed the data. JH and AX wrote the manuscript. All authors read and approved the final manuscript. $\mathrm{JH}$ and $\mathrm{XC}$ confirm the authenticity of all the raw data.

\section{Ethics approval and consent to participate}

Not applicable.

\section{Patient consent for publication}

Not applicable.

\section{Competing interests}

The authors declare that they have no competing interests.

\section{References}

1. Hoyte FCL and Nelson HS: Recent advances in allergic rhinitis. F1000Res 7: F1000 Faculty Rev-1333, 2018.

2. Dávila I, Mullol J, Ferrer M, Bartra J, del Cuvillo A, Montoro J, Jáuregui I, Sastre J and Valero A: Genetic aspects of allergic rhinitis. J Investig Allergol Clin Immunol 19 (Suppl 1): S25-S31, 2009.
3. Incorvaia C, Cavaliere C, Frati F and Masieri S: Allergic rhinitis. J Biol Regul Homeost Agents 32 (Suppl 1): S61-S66, 2018.

4. Numminen J: Allergic rhinitis. Duodecim 133: 473-478, 2017.

5. Brożek JL, Bousquet J, Agache I, Agarwal A, Bachert C, Bosnic-Anticevich S, Brignardello-Petersen R, Canonica GW, Casale T, Chavannes NH, et al: Allergic rhinitis and its impact on asthma (ARIA) guidelines-2016 revision. J Allergy Clin Immunol 140: 950-958, 2017.

6. Bao H, Si D, Gao L, Sun H, Shi Q, Yan Y, Damchaaperenlei D, $\mathrm{Li} \mathrm{C}, \mathrm{Yu} \mathrm{M}$ and Li Y: Acupuncture for the treatment of allergic rhinitis: A systematic review protocol. Medicine (Baltimore) 97: e13772, 2018.

7. Meltzer EO: Allergic rhinitis: Burden of illness, quality of life, comorbidities, and control. Immunol Allergy Clin North Am 36: 235-248, 2016.

8. Gampe N, Darcsi A, Lohner S, Béni S and Kursinszki L: Characterization and identification of isoflavonoid glycosides in the root of Spiny restharrow (Ononis spinosa L.) by HPLC-QTOF-MS, HPLC-MS/MS and NMR. J Pharm Biomed Anal 123: 74-81, 2016.

9. Ong SKL, Shanmugam MK, Fan L, Fraser SE, Arfuso F, Ahn KS, Sethi G and Bishayee A: Focus on formononetin: Anticancer potential and molecular targets. Cancers (Basel) 11: 611, 2019.

10. Yao JN, Zhang XX, Zhang YZ, Li JH,Zhao DY, Gao B, Zhou HN, Gao SL and Zhang LF: Discovery and anticancer evaluation of a formononetin derivative against gastric cancer SGC7901 cells. Invest New Drugs 37: 1300-1308, 2019.

11. Zhang J, Liu L, Wang J, Ren B, Zhang L and Li W: Formononetin, an isoflavone from Astragalus membranaceus inhibits proliferation and metastasis of ovarian cancer cells. J Ethnopharmacol 221: 91-99, 2018

12. Zhang L, Gong Y, Wang S and Gao F: Anti-colorectal cancer mechanisms of formononetin identified by network pharmacological approach. Med Sci Monit 25: 7709-7714, 2019.

13. Yi L, Cui J, Wang W, Tang W, Teng F, Zhu X, Qin J, Wuniqiemu T, Sun J, Wei Y and Dong J: Formononetin attenuates airway inflammation and oxidative stress in murine allergic asthma. Front Pharmacol 11: 533841, 2020.

14. Yuan W, Chen Y, Zhou Y, Bao K, Yu X, Xu Y, Zhang Y, Zheng J, Jiang $\mathrm{G}$ and Hong M: Formononetin attenuates atopic dermatitis by upregulating A20 expression via activation of $\mathrm{G}$ protein-coupled estrogen receptor. J Ethnopharmacol 266: 113397, 2021.

15. Yuan Y, Liu Q, Zhao J, Tang H and Sun J: SIRT1 attenuates murine allergic rhinitis by downregulated HMGB 1/TLR4 pathway. Scand J Immunol 87: e12667, 2018.

16. Piao CH, Fan YJ, Nguyen TV, Song $\mathrm{CH}$ and Chai $\mathrm{OH}$ : Mangiferin alleviates ovalbumin-induced allergic rhinitis via Nrf2/HO-1/NF- $\mathrm{BB}$ signaling pathways. Int J Mol Sci 21: 3415, 2020.

17. Yang S, Wei L, Xia R, Liu L, Chen Y, Zhang W, Li Q, Feng K, $\mathrm{Yu} \mathrm{M}$, Zhang W, et al: Formononetin ameliorates cholestasis by regulating hepatic SIRT1 and PPAR $\alpha$. Biochem Biophys Res Commun 512: 770-778, 2019.

18. Wills-Karp M: Interleukin-13 in asthma pathogenesis. Immunol Rev 202: 175-190, 2004.

19. Matsukura S, Stellato C, Georas SN, Casolaro V, Plitt JR, Miura K, Kurosawa S, Schindler U and Schleimer RP: Interleukin-13 upregulates eotaxin expression in airway epithelial cells by a STAT6-dependent mechanism. Am J Respir Cell Mol Biol 24: 755-761, 2001.

20. Wang B, Gao Y, Zheng G, Ren X, Sun B, Zhu K, Luo H, Wang Z and Xu M: Platycodin D inhibits interleukin-13-induced the expression of inflammatory cytokines and mucus in nasal epithelial cells. Biomed Pharmacother 84: 1108-1112, 2016.

21. Xu N and An J: Formononetin ameliorates mast cell-mediated allergic inflammation via inhibition of histamine release and production of pro-inflammatory cytokines. Exp Ther Med 14: 6201-6206, 2017.

22. Wang Y, Che J, Zhao H, Tang J and Shi G: Paeoniflorin attenuates oxidized low-density lipoprotein-induced apoptosis and adhesion molecule expression by autophagy enhancement in human umbilical vein endothelial cells. J Cell Biochem 120: 9291-9299, 2019

23. Jia M, Chen X, Liu J and Chen J: PTEN promotes apoptosis of $\mathrm{H} 2 \mathrm{O} 2$-injured rat nasal epithelial cells through PI3K/Akt and other pathways. Mol Med Rep 17: 571-579, 2018.

24. Sun B, Wang B and Xu M: Esculetin inhibits histamine-induced expression of inflammatory cytokines and mucin in nasal epithelial cells. Clin Exp Pharmacol Physiol 46: 821-827, 2019. 
25. Livak KJ and Schmittgen TD: Analysis of relative gene expression data using real-time quantitative PCR and the 2(-Delta Delta C(T)) method. Methods 25: 402-408, 2001.

26. Kwak S, Choi YS, Na HG, Bae CH, Song SY and Kim YD: Fipronil upregulates inflammatory cytokines and MUC5AC expression in human nasal epithelial cells. Rhinology 58: 66-73, 2020.

27. Shah SA, Ishinaga H, Hou B, Okano M and Takeuchi K: Effects of interleukin-31 on MUC5AC gene expression in nasal allergic inflammation. Pharmacology 91: 158-164, 2013.

28. Wang X, Li Y, Luo D, Wang X, Zhang Y, Liu Z, Zhong N, Wu M and Li G: Lyn regulates mucus secretion and MUC5AC via the STAT6 signaling pathway during allergic airway inflammation. Sci Rep 7: 42675, 2017.

29. Cheng L, Chen J, Fu Q, He S, Li H, Liu Z, Tan G, Tao Z, Wang D, Wen W, et al: Chinese society of allergy guidelines for diagnosis and treatment of allergic rhinitis. Allergy Asthma Immunol Res 10: 300-353, 2018.

30. Kakli HA and Riley TD: Allergic rhinitis. Prim Care 43 465-475, 2016

31. Meng Y, Wang $\mathrm{C}$ and Zhang L: Recent developments and highlights in allergic rhinitis. Allergy 74: 2320-2328, 2019.

32. Schuler Iv CF and Montejo JM: Allergic rhinitis in children and adolescents. Pediatr Clin North Am 66: 981-993, 2019.

33. Noguchi E, Shibasaki M, Arinami T, Takeda K, Maki T, Miyamoto T, Kawashima T, Kobayashi K and Hamaguchi $\mathrm{H}$ : Evidence for linkage between asthma/atopy in childhood and chromosome 5q31-q33 in a Japanese population. Am J Respir Crit Care Med 156: 1390-1393, 1997.

34. Andiappan AK, Wang de Y, Anantharaman R, Suri BK, Lee BT, Rotzschke O, Liu J and Chew FT: Replication of genome-wide association study loci for allergic rhinitis and house dust mite sensitization in an Asian population of ethnic Chinese in Singapore. J Allergy Clin Immunol 131: 1431-1433.e8, 2013.

35. Teng B, Zhang X, Yi C, Zhang Y, Ye S, Wang Y, Tong DQ and $\mathrm{Lu} \mathrm{B}$ : The association between ambient air pollution and allergic rhinitis: Further epidemiological evidence from Changchun, Northeastern China. Int J Environ Res Public Health 14: 226, 2017.

36. Li CW, Chen DH DH, Zhong JT, Lin ZB, Peng H, Lu HG, Yang Y, Yin J and Li TY: Epidemiological characterization and risk factors of allergic rhinitis in the general population in Guangzhou City in China. PLoS One 9: e114950, 2014.

37. Akhouri S, House SA and Doerr C: Allergic rhinitis (nursing), In: StatPearls. Treasure Island (FL), 2021

38. Bernstein DI, Schwartz G and Bernstein JA: Allergic rhinitis: Mechanisms and treatment. Immunol Allergy Clin North Am 36 261-278, 2016

39. Wang XS, Guan SY, Liu A, Yue J, Hu LN, Zhang K, Yang LK, Lu L, Tian Z, Zhao MG and Liu SB: Anxiolytic effects of formononetin in an inflammatory pain mouse model. Mol Brain 12: 36, 2019.

40. Tay KC, Tan LT, Chan CK, Hong SL, Chan KG, Yap WH, Pusparajah P, Lee LH and Goh BH: Formononetin: A review of its anticancer potentials and mechanisms. Front Pharmacol 10: $820,2019$.

41. Li L, Wang Y, Wang X, Tao Y, Bao K, Hua Y, Jiang G and Hong M: Formononetin attenuated allergic diseases through inhibition of epithelial-derived cytokines by regulating E-cadherin. Clin Immunol 195: 67-76, 2018.

42. Yi L, Lu Y, Yu S, Cheng Q and Yi L: Formononetin inhibits inflammation and promotes gastric mucosal angiogenesis in gastric ulcer rats through regulating NF-kB signaling pathway. J Recept Signal Transduct Res: Oct 26, 2020 (Epub ahead of print).

43. Taylor-Clark T: Histamine in allergic rhinitis. Adv Exp Med Biol 709: 33-41, 2010

44. Hanzlikova J, Sedlacek D, Liska M, Gorcikova J, Vlas T, Amiramini S, Panzner P and Maly M: Histamine increases the level of IFN $\gamma$ produced by HIV-1 specific CTLs and this production depends on total IgE level. J Immunol Methods 375: 1-6, 2012.

45. Gould HJ and Sutton BJ: IgE in allergy and asthma today. Na Rev Immunol 8: 205-217, 2008.

46. Barni S, Liccioli G, Sarti L, Giovannini M, Novembre E and Mori F: Immunoglobulin E ( IgE)-mediated food allergy in children: Epidemiology, pathogenesis, diagnosis, prevention, and management. Medicina (Kaunas) 56: 111, 2020.

47. Bayar Muluk N, Bafaqeeh SA and Cingi C: Anti-IgE treatment in allergic rhinitis. Int J Pediatr Otorhinolaryngol 127: 109674, 2019.

48. Fireman P: Cytokines and allergic rhinitis. Allergy Asthma Proc 17: 175-178, 1996
49. Ohkubo K, Ikeda M, Pawankar R, Gotoh M, Yagi T and Okuda M: Mechanisms of IL-6, IL-8, and GM-CSF release in nasal secretions of allergic patients after nasal challenge. Rhinology 36: 156-161, 1998

50. Peric A, Spadijer-Mirkovic C, Matkovic-Jozin S, Jovancevic L and Vojvodic D: Granulocyte-macrophage colony-stimulating factor production and tissue eosinophilia in chronic rhinitis. Int Arch Otorhinolaryngol 20: 364-369, 2016.

51. López DE and Ballaz SJ: The role of brain cyclooxygenase-2 (Cox-2) beyond neuroinflammation: Neuronal homeostasis in memory and anxiety. Mol Neurobiol 57: 5167-5176, 2020.

52. Cui J and Jia J: Natural COX-2 inhibitors as promising anti-inflammatory agents: An update. Curr Med Chem 28: 3622-3646, 2021

53. Li Z, Dong X, Zhang J, Zeng G, Zhao H, Liu Y, Qiu R, Mo L and Ye Y: Formononetin protects TBI rats against neurological lesions and the underlying mechanism. J Neurol Sci 338: 112-117, 2014.

54. Aladaileh SH, Hussein OE, Abukhalil MH, Saghir SAM, Bin-Jumah M, Alfwuaires MA, Germoush MO, Almaiman AA and Mahmoud AM: Formononetin upregulates Nrf2/HO-1 signaling and prevents oxidative stress, inflammation, and kidney injury in methotrexate-induced rats. Antioxidants (Basel) 8: 430, 2019.

55. Pradère JP, Hernandez C, Koppe C, Friedman RA, Luedde T and Schwabe RF: Negative regulation of NF- $\mathrm{BB}$ p65 activity by serine 536 phosphorylation. Sci Signal 9: ra85, 2016.

56. Huh JE, Lee WI, Kang JW, Nam D, Choi DY, Park DS, Lee SH and Lee JD: Formononetin attenuates osteoclastogenesis via suppressing the RANKL-induced activation of NF- $\mathrm{BB}$, c-Fos, and nuclear factor of activated T-cells cytoplasmic 1 signaling pathway. J Nat Prod 77: 2423-2431, 2014.

57. Liao L, Huang L, Wei X, Yin L, Wei X and Li T: Bioinformatic and biochemical studies of formononetin against liver injure. Life Sci 272: 119229, 2021.

58. Okuda K, Chen G, Subramani DB, Wolf M, Gilmore RC, Kato T, Radicioni G, Kesimer M, Chua M, Dang H, et al: Localization of secretory mucins MUC5AC and MUC5B in normal/healthy human airways. Am J Respir Crit Care Med 199: 715-727, 2019.

59. Zhang YZ, Wu QJ, Yang X, Xing XX, Chen YY and Wang H: Effects of SIRT1/Akt pathway on chronic inflammatory response and lung function in patients with asthma. Eur Rev Med Pharmacol Sci 23: 4948-4953, 2019

60. Wu Z, Chen J, Zhao W, Zhuo CH and Chen Q: Inhibition of miR-181a attenuates sepsis-induced inflammation and apoptosis by activating Nrf2 and inhibiting NF- $\mathrm{KB}$ pathways via targeting SIRT1. Kaohsiung J Med Sci 37: 200-207, 2021.

61. Oza MJ and Kulkarni YA: Formononetin ameliorates diabetic neuropathy by increasing expression of SIRT1 and NGF. Chem Biodivers 17: e2000162, 2020.

62. Hwang JS, Kang ES, Han SG, Lim DS, Paek KS, Lee CH and Seo HG: Formononetin inhibits lipopolysaccharide-induced release of high mobility group box 1 by upregulating SIRT1 in a PPARס-dependent manner. PeerJ 6: e4208, 2018

63. Li J, Wang B, Luo Y, Zhang Q, Bian Y and Wang R: Resveratrol-mediated SIRT1 activation attenuates ovalbumin-induced allergic rhinitis in mice. Mol Immunol 122: $156-162,2020$.

64. Niu Y, Wang J, Li Z, Yao K, Wang L and Song J: HIF1 $\alpha$ deficiency in dendritic cells attenuates symptoms and inflammatory indicators of allergic rhinitis in a SIRT1-dependent manner. Int Arch Allergy Immunol 181: 585-593, 2020.

65. Van Nguyen T, Piao CH, Fan YJ, Shin DU, Kim SY, Song HJ, Song $\mathrm{CH}$, Shin $\mathrm{HS}$ and Chai OH: Anti-allergic rhinitis activity of $\alpha$-lipoic acid via balancing Th17/Treg expression and enhancing Nrf2/HO-1 pathway signaling. Sci Rep 10: 12528, 2020.

66. Jung KA and Kwak MK: The Nrf2 system as a potential target for the development of indirect antioxidants. Molecules 15: 7266-7291, 2010

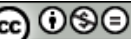

This work is licensed under a Creative Commons Attribution-NonCommercial-NoDerivatives 4.0 International (CC BY-NC-ND 4.0) License. 